\title{
Advances in silicon photonics segmented electrode Mach-Zehnder modulators and peaking enhanced resonant devices
}

S. Sharif Azadeh, J. Müller, F. Merget, S. Romero-García, B. Shen, et al.

S. Sharif Azadeh, J. Müller, F. Merget, S. Romero-García, B. Shen, J. Witzens, "Advances in silicon photonics segmented electrode Mach-Zehnder modulators and peaking enhanced resonant devices," Proc. SPIE 9288, Photonics North 2014, 928817 (25 September 2014); doi: $10.1117 / 12.2075836$

SPIE. Event: Photonics North 2014, 2014, Montréal, Canada 


\title{
Advances in Silicon Photonics Segmented Electrode Mach-Zehnder Modulators and Peaking Enhanced Resonant Devices
}

\author{
S. Sharif Azadeh*, J. Müller, F. Merget, S. Romero-García, B. Shen, J. Witzens \\ Integrated Photonics Laboratory, RWTH Aachen University, \\ Sommerfeldstraße 24, 52074 Aachen, Germany
}

\begin{abstract}
We report recent progress made in our laboratory on travelling wave Mach-Zehnder Interferometer based Silicon Photonics modulators with segmented transmission lines, as well as on resonant ring modulators and add-drop multiplexers with peaking enhanced bandwidth extended beyond the photon lifetime limit. In our segmented transmission lines, microstructuring of the electrodes results in radio-frequency modes significantly deviating from the transverse electromagnetic (TEM) condition and allows for additional design freedom to jointly achieve phase matching, impedance matching and minimizing resistive losses. This technique was found to be particularly useful to achieve the aforementioned objectives in simple back-end processes with one or two metallization layers. Peaking results from intrinsic time dynamics in ring resonator based modulators and add-drop multiplexers and allows extending the bandwidth of the devices beyond the limit predicted from the photon lifetime. Simple closed form expressions allow incorporating peaking into system level modeling.
\end{abstract}

Keywords: Electro-Optical Modulation, Silicon Photonics, Integrated Optics, Optical Interconnects.

\section{INTRODUCTION}

Ongoing growth of cloud computing is creating significant challenges for interconnect systems in data centers and high performance computing systems mainly in terms of data-rate, power consumption, size and cost [1]. Emerging short range interconnect solutions are required to feature reduced power consumption and to be more cost efficient while supporting higher data rates. Aggressive cost targets have for example been set at below \$1/Gpbs [2]. With serial 25 Gbps Infiniband EDR systems already in production, optical interconnects have become an essential technology enabling the scaling of data centers. Maturing Silicon Photonics (SiP) technology allowing mass production of planar integrated photonics at a competitive cost due to the utilization of existing CMOS infrastructure [3] and enabling high integration of optical devices such as modulators [4] and photodetectors [5] at the waferscale is expected to be particularly competitive to service an emerging need for extended reach single-mode high-speed data center interconnects in the $500 \mathrm{~m}-2 \mathrm{~km}$ range [6].

Tremendous efforts have been made in recent years to maximize the bandwidth and reduce the power consumption of SiP modulators. Electro-optical modulation based on the free carrier dispersion effect in reverse biased pn or pin junctions has yielded promising results both in terms of high speed operation (up to several tens of $\mathrm{GHz}$ ) and low power consumption [7]. Several schemes can be chosen from to electrically drive such structures that can be broadly categorized into 1 . driving the modulator as a lumped element with a monolithically or hybridly co-integrated driver, e.g. as is typically done for resonant ring modulators (RRMs) [8] or very short Mach Zehnder Modulators (MZIs), 2. impedance matched driving of travelling wave (TW) modulators [9] and 3. driving of segmented modulators with a cointegrated distributed driver [10].

For TW MZIs, difficulties in achieving high bandwidth operation arise from RF losses of the loaded transmission line $[11,12]$ and from phase mismatch between the light and the travelling RF wave. Moreover, the impedance mismatch between the driver (or $50 \Omega$ transmission line) and the modulator should be minimized in order to avoid RF power backreflections and the resulting reduction of modulation efficiency. The lumped element configuration is beneficial in terms of RF power consumption [13] but requires the modulator to be very small and thus keeping high modulation efficiency and high extinction can be challenging for linear modulators. Increasing the modulation efficiency in compact depletion

*ssharif@iph.rwth-aachen.com; phone +49 24180 20027; fax +49 24180 22212; iph.rwth-aachen.de

Photonics North 2014, edited by Steve MacLean, David V. Plant, Proc. of SPIE Vol. 9288, 928817

(C) 2014 SPIE · CCC code: 0277-786X/14/\$18 · doi: 10.1117/12.2075836 
modulators can be achieved by increasing the phase tuners' capacitance [8], by utilizing resonant enhancement in ring modulators [14] or by leveraging slow-light effects in photonic crystal waveguides [15]. RRMs benefit from a small footprint and combine electro-optic modulation with frequency selective operation, making them well suited for wavelength division multiplexed (WDM) systems. The cut-off frequency is limited by the quality factor (Q-factor) of the ring and the resulting photon lifetime as well as by the RC time constant of the phase shifter [16]. The bandwidth can be increased by designing a smaller Q-factor at the cost of a higher drive voltage required to maintain the same modulation efficiency.

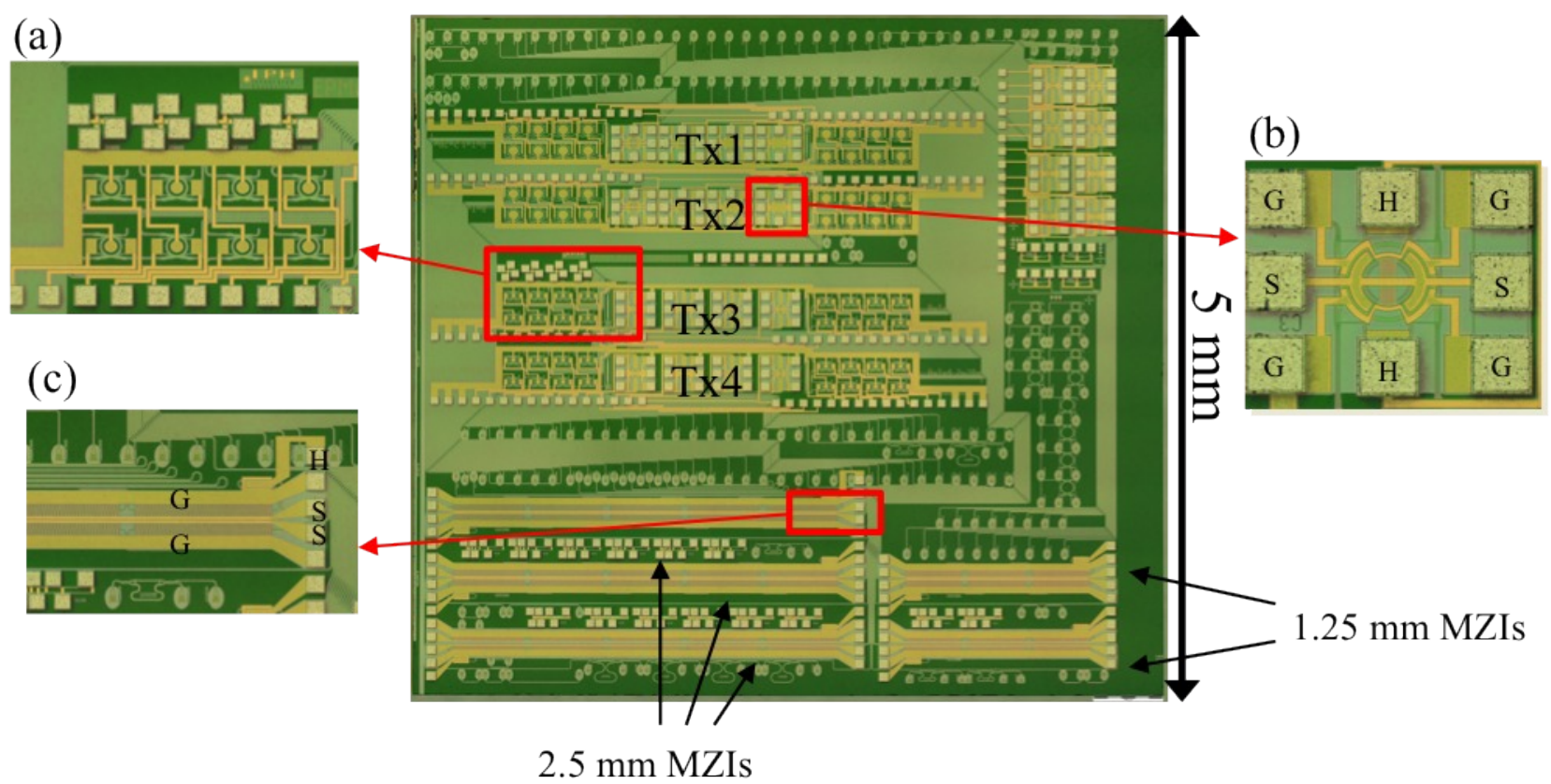

Fig. 1: Microscope image of the chip: (a) Add-drop ring based multiplexers (Rx subsystem): four sets are implemented each combining eight rings with four different Q-factors. (b) Ring modulators: four sets are implemented each consisting of four rings with different Q-factors. The RRMs can be electrically connected to Ground-Signal-Ground (GSG) high speed probes and dispose of a heater $(\mathrm{H})$ for biasing. (c) MZI modulators: two sets of MZIs $(2.5 \mathrm{~mm}$ and $1.25 \mathrm{~mm}$ long) are implemented. The RF driving scheme is Ground-Signal-Signal-Ground (GSSG). For low-speed fine-tuning of the modulators, heaters (H) are embedded (shared ground).

In this work, we describe a method to increase the bandwidth of linear TW MZI modulators while maintaining impedance matching based on segmented or slow wave electrodes. We also describe peaking effects in the electro-optic transfer function of RRMs and the signal transfer function of ring based add-drop multiplexers. This allows enhancing the modulation efficiency-bandwidth product of RRMs by properly choosing the detuning between the resonance of the device and the optical carrier wavelength. Further detuning of the resonance also allows trading-off modulation efficiency and bandwidth, allowing for a dynamic reconfiguration of the device characteristics. Figure 1 shows a microscope image of the chip in which the ring modulators, add-drop filters and MZI modulators were fabricated.

Improvement of the bandwidth of impedance matched travelling wave MZI modulators will be demonstrated utilizing phase-matched slow wave transmission lines in the second section. In the third section, we model and experimentally characterize peaking in RRMs and ring based add-drop multiplexers and demonstrate dynamic reconfiguration of the device characteristics. 


\section{SEGMENTED ELECTRODE MACH ZEHNDER MODULATORS}

\subsection{Introduction}

Reduction of the drive voltage of TW MZI modulators can be accomplished by increasing their length. This, however, requires accurate phase matching and low RF transmission line losses. Another path lies in the increase of the junction capacitance and the phase shifter's efficiency. Both can however be challenging to accomplish while maintaining impedance matching. Electrical signal loss in the transmission line can be the main limiting factor of TW modulator bandwidth [11]. Since it arises primarily out of the resistive losses inside the silicon connecting the metal electrodes with the pn junction, it is a function of transmission line geometry as constrained by impedance matching requirements. As described below, these difficulties become particularly challenging in the case of highly doped, high capacitance pn phase shifters and will be addressed with segmented transmission line designs.

\subsection{Theoretical model: Segmented and slow wave transmission line concept}

In order to design a transmission line well suited for TW modulators, impedance matching and phase matching must be jointly satisfied. Phase matching is independently addressed here by regularly placed optical delay lines (fig. 2(e)) [9] or meandered waveguides [10] that can compensate phase mismatch provided the group index of the waveguides is smaller than the RF index of the transmission line. In silicon-on-insulator (SOI) waveguides, due to the high refractive index contrast between the core and the cladding, small bends can be implemented with small losses and thus the design of phase recovery loops is straightforward. We included such recovery loops, increasing the optical path and equalizing the group delay of the optical path to the phase delay of the transmission line. In our design, the simulated group index of the optical signal $\mathrm{n}_{\mathrm{g}}$ is 3.78 and the RF index of the transmission line $\mathrm{n}_{\mathrm{RF}}$ is 5.6 at $15 \mathrm{GHz}$ (fig. 2(d)). Accordingly, 5 recovery loops were included in the modulator design with a length sized to compensate for this group velocity mismatch (a micrograph of one of them is shown in fig. 2(e)).
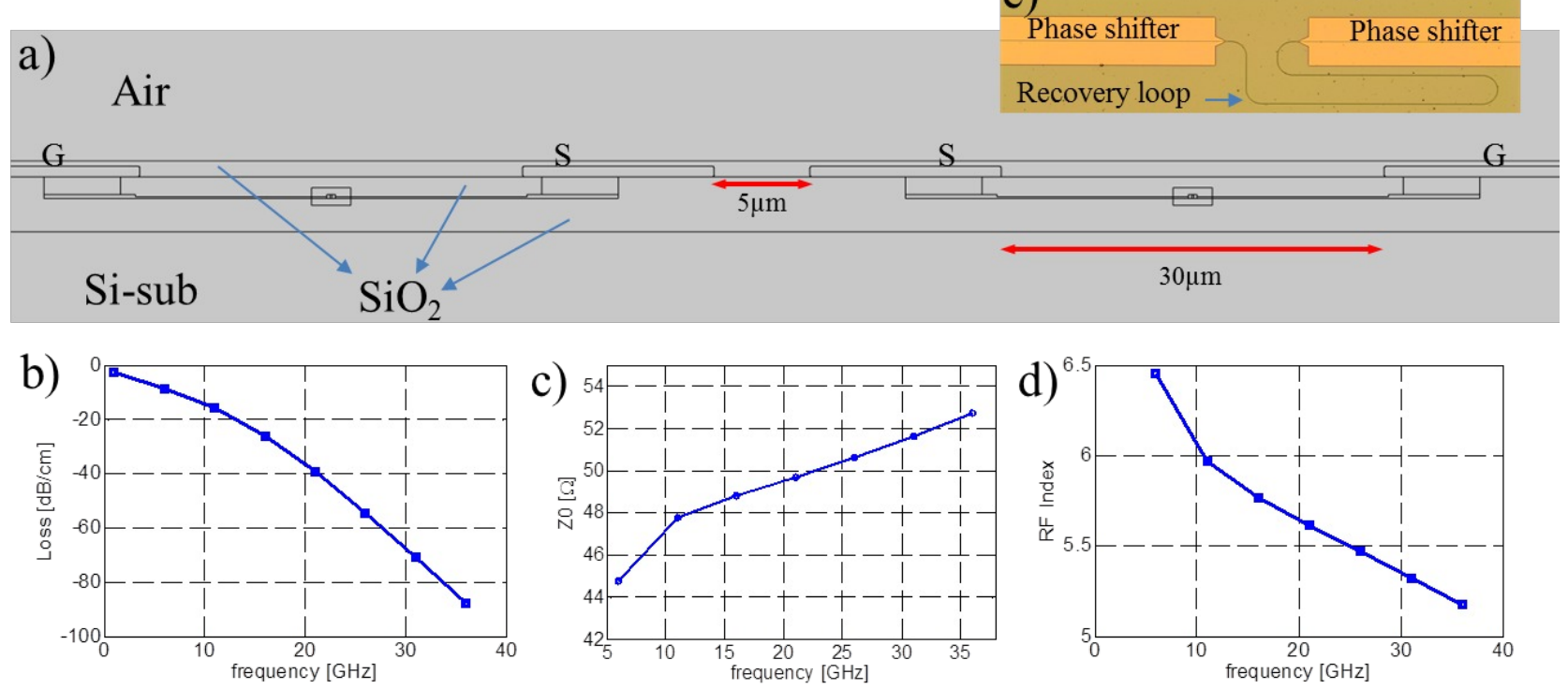

Fig 2: (a) Schematic of the transmission line cross section used in RF simulations for calculation of (b) RF losses, (c) characteristic impedance, and (d) RF index of the transmission line. (e) Microscope image of a recovery loop.

Since phase matching is separately addressed, the transmission line design is primarily constrained by impedance matching. Design parameters such as electrode spacing (primary parameter) or electrode width (secondary parameter) determine the impedance of the loaded transmission line in simple, non-segmented geometries.

In order to motivate segmented and slow wave transmission lines, we will first describe the impedance matching problem with help of a simplified analytical model. While complex travelling wave structures as described here should be simulated in order to obtain accurate results (and were actually designed with full electromagnetic simulations), the simplified analytical models described in this section exemplify the design tradeoffs motivating the introduction of 
segmented and slow wave transmission line structures. Since the waveguide primarily adds a capacitive load to the transmission line [11], the characteristic impedance can be described as

$$
Z_{T L}=\sqrt{\frac{L_{p}}{C_{p}+C_{w g}}}
$$

where $L_{p}$ and $C_{p}$ are given by the metal electrode layout and respectively correspond to the linear inductance and capacitance of the unloaded transmission line, and $C_{w g}$ is the additional linear capacitive load introduced by the silicon waveguide. While $L_{p}$ and $C_{p}$ cannot be modified independently by changing the electrode geometry (when $C_{p}$ is increased, $L_{p}$ is decreased and vice-versa), $C_{w g}$ is, approximately, simply an additive term given by the pn junction characteristics. We introduce the RF index $n_{U}$ of the unloaded transmission line, i.e., of the transmission line after removing the pn-junction and its capacitive load. It can be written as

$$
n_{U}=c_{0} \sqrt{L_{p} C_{p}}
$$

where $c_{0}$ is the speed of light in vacuum. Since in the absence of segmentation the unloaded transmission line supports a quasi-TEM mode (quasi due to the asymmetry of the cladding materials), $n_{U}$ is a weighted average of the RF indices of the cladding materials and is simulated as $\sim 1.8$, in between the $\mathrm{RF}$ index of $\mathrm{SiO}_{2}$ (the bottom cladding material) and air (the top cladding material) for the types of structures reported here. Incorporating (2) in (1) we get

$$
Z_{T L}=\frac{n_{U}}{c_{0}} \frac{1}{\sqrt{C_{p}} \sqrt{C_{p}+C_{w g}}}<\frac{n_{U}}{c_{0}} \frac{1}{\sqrt{C_{p}} \sqrt{C_{w g}}}
$$

This clearly shows that in order to maintain impedance matching (e.g. keeping $Z_{T L}=50 \Omega$ ) the capacitance of the unloaded transmission line should be decreased when the waveguide doping is increased, the latter first changed in order to increase the junction capacitance and thus the modulation efficiency. This can be achieved by increasing the distance between the signal and ground lines. It means that for higher waveguide doping the metal lines need to be spaced more, leading to extended fringe fields and more RF interference, potentially more RF losses resulting from more interaction between the electromagnetic wave with the environment, and most importantly larger RF losses due to a higher phase shifter series resistance resulting from the long signal path in silicon. For the designs reported in the next section the typical spacing between signal and ground lines is for example on the order of $30 \mu \mathrm{m}$, a long distance over which to transmit the signal through the relatively high resistivity doped silicon used for electrical connectivity between the waveguides and the transmission line electrodes (reducing this resistivity by e.g. silicidation does not necessarily reduce the RF losses as it can also be accompanied by increasing longitudinal electrical currents in the silicon region [11]). As a refinement to address these difficulties, finger extensions as shown in fig. 3(a) can be introduced between the electrodes and the waveguide. These provide a low resistivity path between the electrode and the waveguide, while at the same time minimally perturbing the linear capacitance and inductance of the structure, thus not affecting the transmission line impedance. In this design, the extensions were purposefully implemented relatively sparsely $(10 \mu \mathrm{m}$ spacing between adjacent extensions) and offset relative to one another so as to minimize the additional capacitance. Since the current flows within the extension perpendicular to the main axis of the transmission line, the introduced additional selfinductance is minimal and the quasi-TEM symmetry broken even in the unloaded transmission line. The reduced series resistance of the phase shifter lowers the RF propagation losses and improves the bandwidth of the modulator. Details on the design of these structures can be found in [9].

As mentioned above, we introduced the finger extensions so as to minimally perturb the linear capacitance and inductance of the loaded transmission line. Another extreme can be implemented in which the excess capacitance introduced by the extensions is maximized while the linear inductance maintained at the level predicted from the larger spacing between the main signal and ground lines. This is for example the case for the T-shaped extensions shown in fig. 3(b). (Equivalently, one could say that the linear inductance is increased while the linear capacitance maintained at the level given by the spacing between opposing $\mathrm{T}$ shaped elements - this is merely a matter of how one defines the baseline structure. The bottom line is simply that the product of the two quantities is increased beyond the value that would be expected from the quasi-TEM mode of a non-segmented TL). To the first order, this is equivalent to adding additional additive capacitive loading, further slowing down the RF wave and further exacerbating the problem described by eq. 3 : The excess capacitance of the T-extensions can be lumped to the waveguide capacitance in the formula. The spacing between the main signal lines will thus have to be further increased (or the width of the signal lines decreased). Adding $\mathrm{T}$-junctions however is very useful for devices in which the RF propagation velocity in the transmission line would 
otherwise be higher than the group velocity of the waveguide, a situation where phase matching via optical delay loops would not work since negative delay loop lengths would be required. In that situation, further slowing down the RF mode with a slow wave electrode (SWE) design allows recovering phase matching, a technique that has been extensively applied to GaAs modulators [17,18] and more recently to SiP modulators $[19,20]$. Typically, segmented TL electrodes as shown in fig. 3(a) will be useful for highly doped, high-capacitance phase shifters for which the phase velocity of the TL is low and impedance matching to high impedances difficult, while slow wave TL of the type shown in fig. 3(b) might be most useful for lower doping levels, for which impedance matching to $50 \Omega$ is less of a problem, but achieving phase matching might require slowing down the RF mode. For example, in section 2.3 we will compare recent results for modulators implemented in the IMEC process with pn-junction doping levels $\mathrm{n}-=1 \mathrm{e} 18 \mathrm{~cm}^{-3}$ and $\mathrm{p}-=1 \mathrm{e} 18 \mathrm{~cm}^{-3}$ with similar, previously published modulators optimized for and fabricated in the IME process $\left(\mathrm{n}-=1.3 \mathrm{e} 17 \mathrm{~cm}^{-3}\right.$ and $\mathrm{p}-=$ $4 \mathrm{e} 17 \mathrm{~cm}^{-3}$ ). While for the modulators fabricated in the IMEC process the delay lines turned out to be properly sized, we erroneously introduced optical delay lines in the devices fabricated at IME, while the propagation velocity of the transmission lines was actually already higher than the group velocity of the waveguides, and SWE electrodes would have been more appropriate. As reported in [9], the modulator bandwidth was thus partially limited by residual phase mismatch.
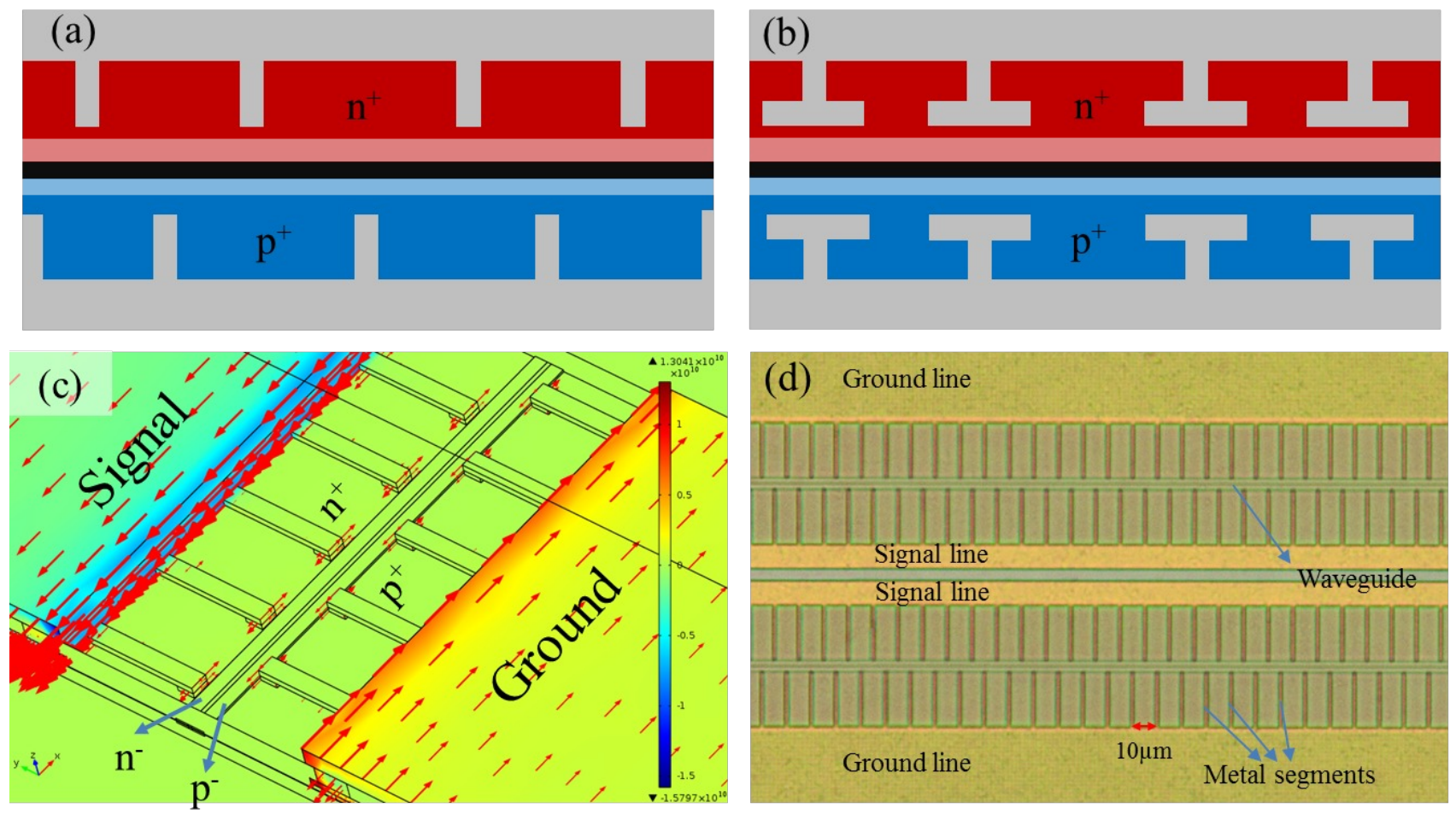

Fig. 3: Schematic of the segmented electrodes in MZIs for (a) segmented electrodes minimizing excess capacitance and (b) slow-wave transmission lines (not to scale). (c) 3D model of metal extensions and simulated longitudinal current flow along the main axis of the TL. (d) Microscope image of the implemented segmented electrodes.

\subsection{Experimental results and discussion}

In this section, we describe experimental results obtained with devices fabricated via EpixFab in the IMEC process line (the "high junction capacitance devices" with a linear pn-junction capacitance of $370 \mathrm{pF} / \mathrm{m}$ at $-2 \mathrm{~V}$ bias) and compare them with earlier devices fabricated at IME (the "low junction capacitance devices" with a linear pn-junction capacitance of $230 \mathrm{pF} / \mathrm{m}$ at $-2 \mathrm{~V}$ bias). Since details on the latter are already available in the literature [9], we focus the device description on the former. The high junction capacitance devices were fabricated out of an SOI wafer with a $2 \mu \mathrm{m}$ buried oxide and a $220 \mathrm{~nm}$ thick silicon device layer. The silicon waveguides are cladded with a top oxide layer thicker than 1 $\mu \mathrm{m}$ fabricated with a standard back-end process in the IMEC SiP pilot line. In contrast to our previous design for MZI modulators (the "low junction capacitance modulators"), the doping is increased by a factor three in order to increase the modulation efficiency. 
The RF signal driving scheme is GSSG in order to reduce the crosstalk between the two arms of the MZI as described in [9] and shown in fig. 4. The two central electrodes of the MZI carry the same RF signal (as opposed to complementary signals) and thus do not suffer from electromagnetic interference between them. In order to keep the pn junctions reverse biased, a DC offset is added to the RF signals by means of a bias-T (note that this could also have been accomplished without a bias-T by shifting up the voltage of the leftmost RF ground line to a higher DC level making an integrated solution easier to realize). The high doping of the waveguide resulted in an increased $C_{w g}$ thus making voltage actuated free carrier density modulation more effective. This can result in a suboptimum characteristic impedance below $50 \Omega$ as explained in the previous section unless large coplanar electrode spacing is implemented ( $30 \mu \mathrm{m}$ in this case). $\mathrm{S}_{11}$ measurements confirm that in the whole frequency range up to $50 \mathrm{GHz}$ the RF back reflected power is less than $10 \mathrm{~dB}$.

a)

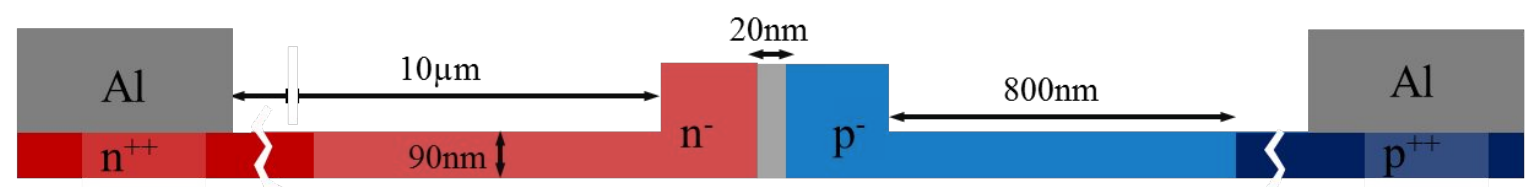

b)

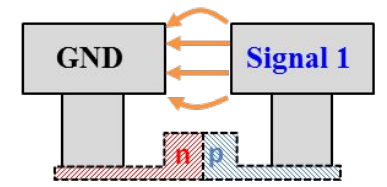

c)

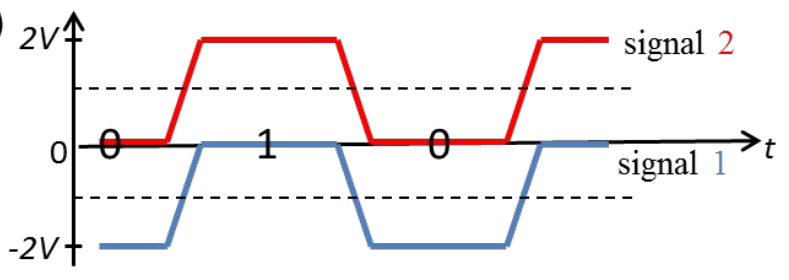

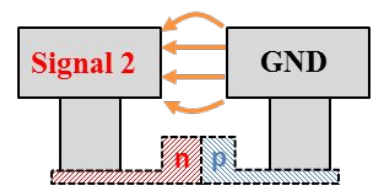

d) Color Coding: E-field Amplitude

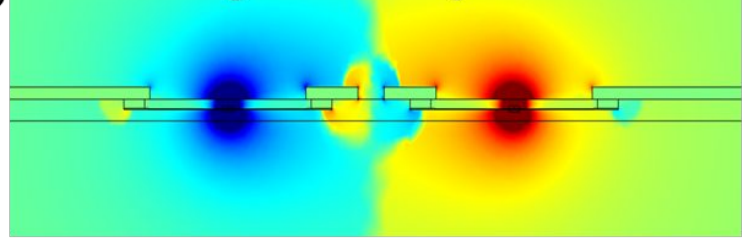

e)

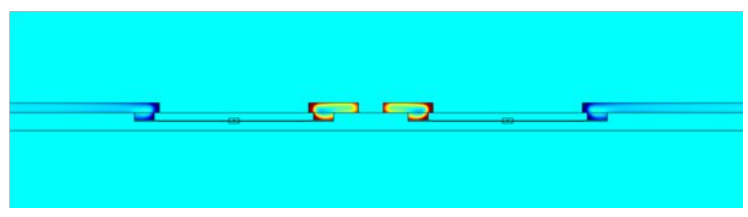

Fig 4: (a) Schematic of the implemented active waveguide, (b) schematic of the GSSG driving scheme and (c) RF driving signals applied to the two arms of the MZI. (d) Simulated E-field in the GSSG driving scheme (e) longitudinal current flow along the main axis of the transmission line at $18 \mathrm{GHz}$. The color-codings respectively represent the sign of the E-field (d) and of the current flow (e).

The waveguide is formed by a deep $130 \mathrm{~nm}$ etch into the $220 \mathrm{~nm}$ silicon layer providing electrical connectivity to the junction. The slabs are highly doped $\left(>10^{20} \mathrm{~cm}^{-3}\right)$ starting $800 \mathrm{~nm}$ away from the waveguide edge in order to ensure a sufficiently low overlap between the optical mode and the highly doped areas and avoid excessive absorption losses (the additional optical loss due to highly doped regions is less than $1 \mathrm{~dB} / \mathrm{cm}$ ).

The "high junction capacitance modulators" were fabricated in two versions, with a $2.5 \mathrm{~mm}$ long and a $1.2 \mathrm{~mm}$ long phase shifter. Figure 5(a) shows the wavelength shift for different applied voltages for a $2.5 \mathrm{~mm}$ long modulator. A $\pi$ phase shift is achieved in single arm configuration when 8 volts (between 0 and -8) are applied to a single arm, which corresponds to a $\mathrm{V} \pi \mathrm{L}$ of $2.0 \mathrm{~V} \cdot \mathrm{cm}$, twice the $\mathrm{V} \pi \mathrm{L}$ of the phase shifter reported in [9] due to the higher doping levels. The on-chip optical insertion loss (that is, excluding grating coupler losses) of the modulator is $6.5 \mathrm{~dB}$ for the long modulator and $4.5 \mathrm{~dB}$ for the shorter modulator. Required dual-drive drive voltages for full extinction are respectively $3.2 \mathrm{~V}$ and $7 \mathrm{~V}$. The $-3 \mathrm{dBe}$ electro-optical cut-off frequency of the modulator $\left(\mathrm{f}_{-3 \mathrm{~dB}}\right)$ is $17 \mathrm{GHz}$ for the long modulator and $23 \mathrm{GHz}$ for the shorter modulator ( $3 \mathrm{~dB}$ cut-off in the electrical convention). The electro-optic $\mathrm{S}_{21}$ of both modulators are shown in figs. 5(b) and 5(c). In principle, assuming the only factor limiting the bandwidth to be the RF transmission line losses (perfect phase matching), $\mathrm{f}_{-3 \mathrm{~dB}}$ is expected to be at the frequency where the electrical power transmitted through the transmission line, $\mathrm{S}_{21, \mathrm{RF}}$, is attenuated by $6.4 \mathrm{~dB}$. Since according to measurements these two points exactly match for the short modulator (at $23 \mathrm{GHz}$ ), we conclude that the main factor limiting this modulator's bandwidth are transmission line losses and that phase matching and impedance matching (confirmed by $\mathrm{S}_{11}$ measurements) are not limiting the bandwidth. Also, for the long modulator, since the $-6.4 \mathrm{~dB}$ point of the RF transmission is at $18 \mathrm{GHz}$, the electro-optic bandwidth reduction resulting from phase mismatch is tiny. These results are exactly fitting predictions of our finite element model (FEM) simulations (the simulated RF losses shown in fig. 2(b) result in a $3 \mathrm{~dB}$ electro-optical bandwidth 
of $23 \mathrm{GHz}$ for the short modulator and $18 \mathrm{GHz}$ for the long modulator at a reverse bias of $2 \mathrm{~V}$ in the absence of any phase mismatch). In this simulation model, the capacitance of the waveguide was set to $370 \mathrm{pF} / \mathrm{m}$ and the series resistance of the waveguide to $8 \mathrm{~m} \Omega \cdot \mathrm{m}$ as calculated by solving the poisson equation and drift-diffusion equation with Synopsys' TCAD tool. In the 2D FEM simulations, the effect of the segmented electrodes is modeled by reducing the resistivity of the highly doped regions allowing the simulation of this $3 \mathrm{D}$ structure with a simpler, computationally efficient $2 \mathrm{D}$ model. This approximation was furthermore validated by performing 3D simulations using Ansoft HFSS. For both the long and short modulators, a gap of $20 \mathrm{~nm}$ between $\mathrm{n}$ - and $\mathrm{p}$ - doped regions inside the waveguide was introduced with the purpose of bandwidth enhancement by reduction of the waveguide capacitance (this was easier to do than modifying the implant densities in an established process). Figure 5(d) shows the measured eye diagrams for the $2.5 \mathrm{~mm}$ long MZI modulator with a drive voltage of $2 \mathrm{~V}$ and a laser output power of $10 \mathrm{~mW}$ at $1541 \mathrm{~nm}$ (no electrical preamplifier or optical power amplifier were used, even though at least $9 \mathrm{~dB}$ of optical power where cumulatively lost at the two fiber to chip interfaces).

a)

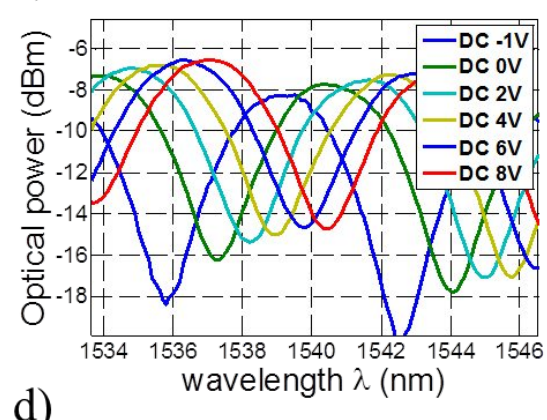

d)

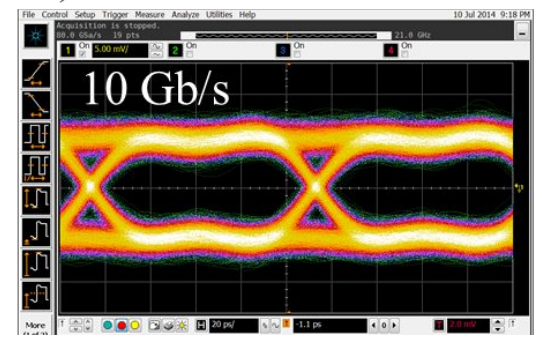

b)
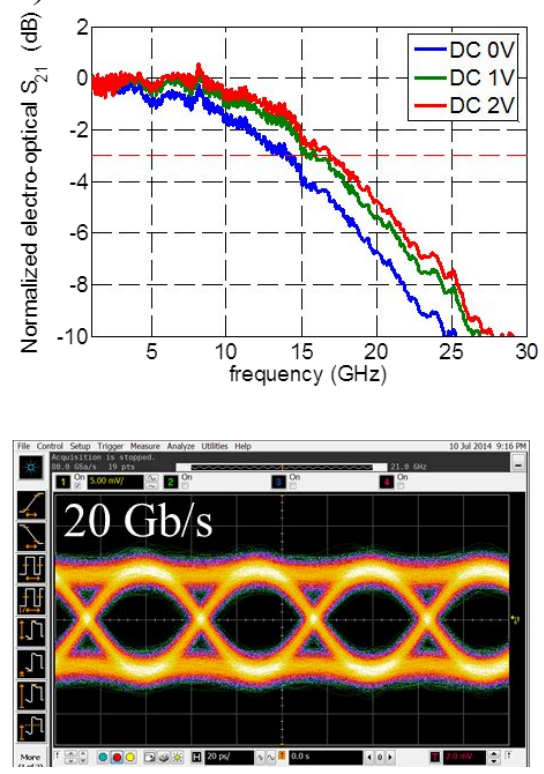

c)
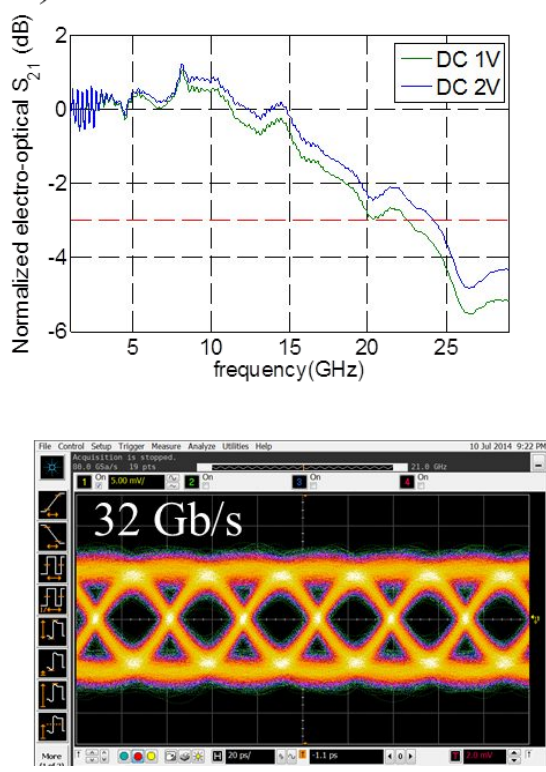

Fig 5: (a) Wavelength shift of the optical transmission for different DC voltages (grating coupler loss was de-embedded), (b) electrooptical $\mathrm{S}_{21}$ of the $2.5 \mathrm{~mm}$ modulator, (c) electro-optical $\mathrm{S}_{21}$ of the $1.25 \mathrm{~mm}$ modulator, and (d) 10, 20 and 32 Gbps eye diagrams (PRBS15) of the $2.5 \mathrm{~mm}$ modulator visualized with a $20 \mathrm{GHz}$ real time oscilloscope.

In contrast to our previously implemented MZI modulators [9], also relying on segmented electrodes, the current device $(2.5 \mathrm{~mm}$ version) has a reduced drive voltage $(3.2 \mathrm{~V}$ versus $4 \mathrm{~V})$, reduced on-chip insertion losses $(6.5 \mathrm{~dB}$ versus $8.8 \mathrm{~dB})$, and a slightly higher bandwidth (17 GHz versus $16.2 \mathrm{GHz})$.

Table 1 summarizes the device performance metrics. The lower $V \pi$ results from higher doping levels $\left(1 \mathrm{e} 18 \mathrm{~cm}^{-3}\right.$ both for $\mathrm{n}$ - and $\mathrm{p}$-), which results in $11 \times 10^{-5}$ effective index change for a $2 \mathrm{~V}$ signal applied to the phase shifter, while in the previous design it was $6 \times 10^{-5}$ for the same applied voltage. Thus, even though the device is shorter, the final phase shift is higher. The reduction of the optical loss is primarily due to the implementation of better coupling devices. While the calculated losses due to free carrier absorption increased from $3.4 \mathrm{~dB}$ to $5 \mathrm{~dB}$ (the increased free carrier losses due to higher implants overcompensate the shortened device length), the excess loss incurred by two multi-mode interferometer (MMI) splitters and excess waveguide losses where less than $1.5 \mathrm{~dB}$ here.

Finally, simulations show that our previous device was penalized by the frequency dependence of the RF transmission line index primarily due to a partial screening of the capacitive waveguide load at high frequencies as the RC bandwidth limit of the phase shifter is approached [11]. Since the intrinsic RC bandwidth limit of the phase shifter was increased from $46 \mathrm{GHz}$ to $54 \mathrm{GHz}$ due to a reduction of the phase shifter's series resistance, TL RF index dispersion was reduced, allowing phase matching to be maintained over a broad frequency range up to the RF loss limited modulator bandwidth. 
Table 1: Comparison between the modulators' characteristics.

\begin{tabular}{|c|c|c|c|c|c|}
\hline & Length & $\begin{array}{c}\text { Optical } \\
\text { Ins. Loss }\end{array}$ & $\begin{array}{c}\text { Vpp for Full } \\
\text { Extinction in } \\
\text { Push-Pull }\end{array}$ & $\begin{array}{c}\text { Modulation Efficiency } \\
\text { (V } \boldsymbol{\pi} \cdot \mathbf{L})\end{array}$ & $\begin{array}{c}\mathbf{3} \text { dBe Electro-Optical } \\
\text { Bandwidth }\end{array}$ \\
\hline $\begin{array}{c}\text { Low Cap. } \\
\text { Mod. [9] }\end{array}$ & $4 \mathrm{~mm}$ & $8.8 \mathrm{~dB}$ & $4 \mathrm{~V}$ & $4.0 \mathrm{~V} \cdot \mathrm{cm}$ & $16.2 \mathrm{GHz}$ \\
\hline $\begin{array}{c}\text { High Cap. } \\
\text { Mod. }\end{array}$ & $2.5 \mathrm{~mm}$ & $6.5 \mathrm{~dB}$ & $3.2 \mathrm{~V}$ & $2.0 \mathrm{~V} \cdot \mathrm{cm}$ & $17 \mathrm{GHz}$ \\
\hline $\begin{array}{c}\text { High Cap. } \\
\text { Mod. }\end{array}$ & $1.25 \mathrm{~mm}$ & $4 \mathrm{~dB}$ & $7 \mathrm{~V}$ & $2.25 \mathrm{~V} \cdot \mathrm{cm}$ & $23 \mathrm{GHz}$ \\
\hline
\end{tabular}

\section{PEAKING ENHANCED RESONANT RING BASED DEVICES}

\subsection{Modeling of peaking effect in ring modulators and add-drop multiplexers}

Recently substantial progress has been made in describing accurately the transient time dynamics of ring resonators and their impact on the electro-optical (EO) $\mathrm{S}_{21}$ parameters of ring based EO modulators. The frequency response of a ring modulator shows an overshoot when the laser is detuned from the ring resonance beyond the point of maximum DC electro-optic response (where the resonance has the steepest slope). Peaking has been observed in experimental studies [21-26] and has been theoretically described in a number of contributions [24-30]. In this section, we will be looking at peaking effects both in RRMs as well as in ring based add-drop multiplexers. Peaking in the small signal response of RRMs can be derived by starting with the differential equations (4) and (5) introduced in [27,29] and applying first-order perturbation theory which results in $[25,26]$

$$
\begin{gathered}
\frac{\partial a}{\partial t}=\left(-i \omega_{r}-\frac{1}{\tau_{a}}\right) a+i \mu E_{I n} \\
E_{\text {Out }}=E_{\text {In }}+i \mu a
\end{gathered}
$$

Here, $a$ is the field within the resonator, $\omega_{r}$ is the angular resonant frequency of the ring, $\tau_{a}$ is the $1 / \mathrm{e}$ decay time of the field amplitude, $\mu$ is the time domain coupling parameter and $E_{I n}$ and $E_{\text {Out }}$ are the amplitudes of the incoming and outgoing waveguide modes. When a drive voltage $\delta V \cdot \sin \left(\omega_{m} t\right)$ is applied, the resonance of the modulator is shifted to $\omega_{r}+\delta \omega_{r} \cdot \sin \left(\omega_{m} t\right)$. The nonlinearity of the transfer function does not have to be taken into account here since we are assuming $\delta \omega_{r}$ to be small and we are applying first order perturbation theory to derive the small signal response of the ring modulator

$$
\left|E_{\text {Out }}\right|^{2}-\left\langle\left|E_{\text {Out }}\right|^{2}\right\rangle=\mu R e\left(\left[\frac{\delta \omega_{r} \bar{a}\left(\overline{E_{\text {In }}}+i \mu \bar{a}\right)^{*}}{-i \omega_{m}-\left(i \omega_{0}-i \omega_{r}\right)+\frac{1}{\tau_{a}}}+\frac{\delta \omega_{r}{ }^{*} \bar{a}^{*}\left(\overline{E_{\text {In }}}+i \mu \bar{a}\right)}{-i \omega_{m}+\left(i \omega_{0}-i \omega_{r}\right)+\frac{1}{\tau_{a}}}\right] e^{-i \omega_{m} t}\right)
$$

where $\omega_{m}$ is the modulation frequency, $\omega_{0}$ is the carrier frequency, $E_{I n}=\overline{E_{I n}} e^{-i \omega_{0} t}$, and $\bar{a} e^{-i \omega_{0} t}$ is the value of $a$ in the absence of modulation voltage, i.e., its average or DC value. It should be noted that $\delta \omega_{r}$ is a complex number, where the imaginary part takes loss modulation into account that necessarily arises from the free carrier index modulation in addition to the effective index change. The loss modulation leads to an asymmetry of the small signal response when comparing laser detunings (the difference between the optical carrier frequency and the resonant frequency of the ring) of the same amplitude but different signs. Figure 6(a) visualizes this effect.

The blue and green spectra represent the transfer function of the ring when its resonance is respectively shifted to smaller wavelengths (blue) and larger wavelengths (green). The two vertical lines (red and black) visualize possible optical carrier frequencies. The blue curve corresponds to a higher free carrier density, and thus to a lower effective index and higher round trip losses. The Q-factor of the blue curve is thus also lower, increasing the linewidth of the resonance and pushing out its left flank to even smaller wavelengths. This then increases the modulation depth for the lower wavelength optical carrier. Conversely, the modulation depth for the higher wavelength optical carrier is reduced. Figure 6(b) shows the corresponding electro-optic $S_{21}$ for the two carrier wavelengths. Their detuning relative to the average 
resonance wavelength of the ring modulator is indicated. As expected, the frequency response for the smaller wavelength (positive frequency detuning) has a higher DC modulation efficiency than the frequency response for the higher wavelength (negative frequency detuning).
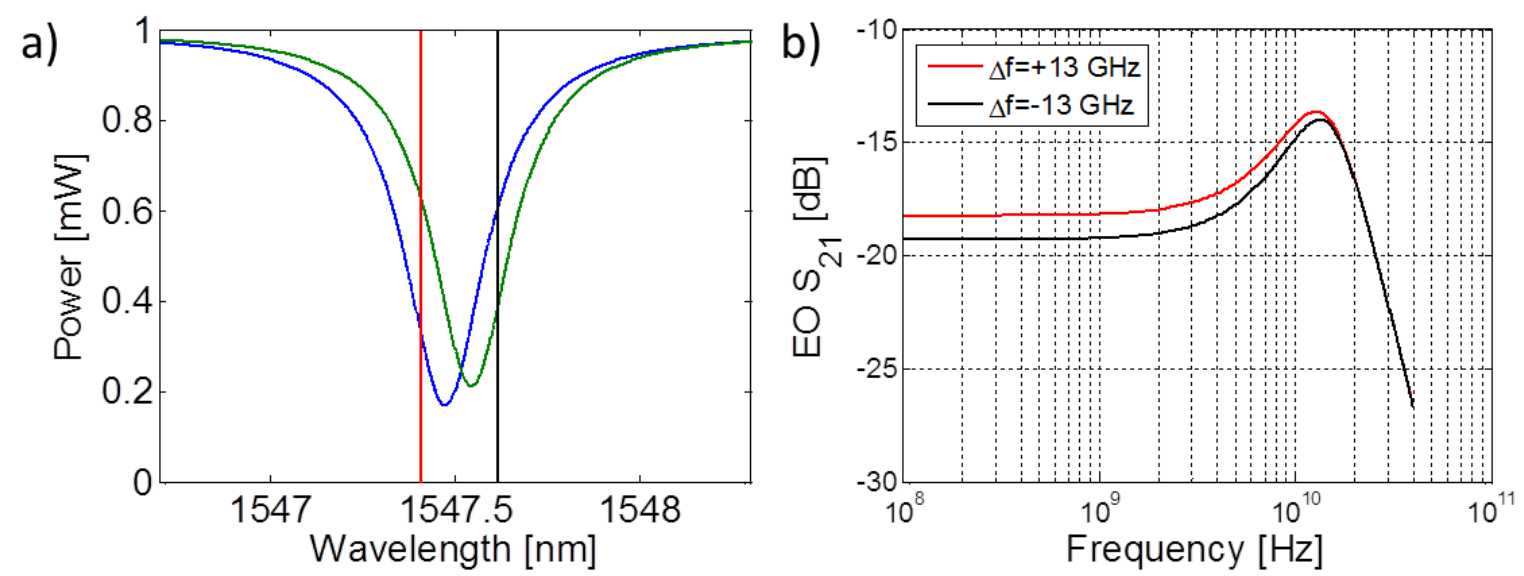

Fig. 6: (a) Optical spectrum for logical 1 and 0 with two possible carrier frequencies indicated by vertical lines. (b) Frequency response for the two indicated carrier frequencies. Even though the two carriers are symmetric relative to the average resonance position of the ring, the resulting $S_{21}$ are different.

Peaking does not only occur in ring modulators, but also in ring based add-drop multiplexers. In order to derive the response of an add-drop multiplexer to an amplitude modulated optical signal, we assume a modulated signal $E_{\text {in }}$ consisting of a carrier and two symmetric side bands that are filtered by a lorentzian filter with amplitude transmission coefficient $\operatorname{tr}(\omega)$. Furthermore, we assume the amplitude of the sidebands to be small relative to the optical carrier (small signal modulation). It should also be noted that the assumption of symmetric sidebands is appropriate when the modulated optical signal is generated by a balanced Mach-Zehnder interferometer operated in push-pull configuration at the $3 \mathrm{~dB}$ point (i.e., a typical low chirp linear modulator). The response of the ring-based add-drop multiplexer is then independent on whether the optical carrier is detuned by the same amount to the right or to the left of the ring resonance (i.e., the $\mathrm{S}_{21}$ of the device is independent on the sign of the detuning). On the other hand, high-speed modulation with a RRM results in asymmetric sidebands as shown in $[25,26]$. This would then also lead to a different add-drop multiplexer response for positive and negative detunings. The add-drop multiplexers where experimentally characterized with an optical signal generated by a low chirp commercial linear modulator (next section), so that the analysis is done here assuming symmetric sidebands

$$
\begin{gathered}
P_{\text {in }}=1+\varepsilon \cdot \cos \left(\omega_{m} t\right) \\
E_{\text {in }}=e^{-i \omega_{0} t}+\frac{\varepsilon}{4} e^{-i\left(\omega_{0}+\omega_{m}\right) t}+\frac{\varepsilon}{4} e^{-i\left(\omega_{0}-\omega_{m}\right) t} \\
\operatorname{tr}(\omega)=\frac{1}{1-i \frac{2 Q\left(\omega-\omega_{r}\right)}{\omega_{r}}} \\
E_{\text {out }}=\operatorname{tr}\left(\omega_{0}\right) e^{-i \omega_{0} t}+\operatorname{tr}\left(\omega_{0}+\omega_{m}\right) \frac{\varepsilon}{4} e^{-i\left(\omega_{0}+\omega_{m}\right) t}+\operatorname{tr}\left(\omega_{0}-\omega_{m}\right) \frac{\varepsilon}{4} e^{-i\left(\omega_{0}-\omega_{m}\right) t}
\end{gathered}
$$

Here, $\varepsilon$ is a small number (terms in $\varepsilon^{2}$ are neglected in the following analysis), $P_{\text {in }}$ is the optical power entering the adddrop multiplexer, $E_{\text {in }}$ is the amplitude entering the cavity, $Q$ is the loaded quality-factor of the cavity, and $E_{\text {out }}$ is the optical amplitude exiting the cavity through the drop port. Note that eq. 9 corresponds to an add-drop multiplexer where the intrinsic $Q$ of the ring is much higher than the loaded $Q$, so that on resonance insertion losses can be neglected. This results in the transmitted optical power at the through port of the add-drop multiplexer to be

$$
\begin{gathered}
P_{\text {out }}=\left|\operatorname{tr}\left(\omega_{0}\right)\right|^{2}+\varepsilon \cdot \operatorname{Re}(T) \cdot \cos \left(\omega_{m} t\right)+\varepsilon \cdot \operatorname{Im}(T) \cdot \sin \left(\omega_{m} t\right) \\
S 21[d B e]=20 \log _{10}(|T|)
\end{gathered}
$$




$$
\begin{gathered}
T=\frac{\operatorname{tr}\left(\omega_{0}\right)^{*} \operatorname{tr}\left(\omega_{0}+\omega_{m}\right)}{2}+\frac{\operatorname{tr}\left(\omega_{0}\right) \operatorname{tr}\left(\omega_{0}-\omega_{m}\right)^{*}}{2} \\
T=\frac{1}{2 \tau_{a}^{2}}\left(\frac{1}{\frac{1}{\tau_{a}}+i\left(\omega_{0}-\omega_{r}\right)} \frac{1}{\frac{1}{\tau_{a}}-i \omega_{m}-i\left(\omega_{0}-\omega_{r}\right)}+\frac{1}{\frac{1}{\tau_{a}}-i\left(\omega_{0}-\omega_{r}\right)} \frac{1}{\frac{1}{\tau_{a}}-i \omega_{m}+i\left(\omega_{0}-\omega_{r}\right)}\right)
\end{gathered}
$$

which takes the same form as eq. (6). As will be shown in the next section, this model is also in excellent agreement with our measurements.

\subsection{Characteristics of the ring modulators and experimental verification of the peaking effect}

RRMs were fabricated in the $220 \mathrm{~nm}$ SOI IMEC pilot line already described in section 2.3. Four different groups of RRMs were defined, labeled as Tx 1 to 4 in the following. The first two groups include rings with a radius of $46 \mu \mathrm{m}$, a waveguide width of $550 \mathrm{~nm}$ and a slab height of $150 \mathrm{~nm}$. The other two groups, Tx 3 and 4, consist of rings with a radius of $28 \mu \mathrm{m}$, a waveguide width of $400 \mathrm{~nm}$ and a slab height of $50 \mathrm{~nm}$ (higher confinement waveguides). For these two groups, a deeper etch depth was necessary in order to implement smaller rings with a larger free spectral range (FSR), at the price of a higher series resistance. The modulation sections of all RRMs are defined with identical pn-junctions operated in reverse bias. Implant concentrations were equally chosen to be $\mathrm{p}^{-}=\mathrm{n}^{-}=1 \mathrm{e} 18 \mathrm{~cm}^{-3}$ for all four groups resulting in an intrinsic Q-factor of 22130 for the rings belonging to groups 1 and 2 and 25530 for the rings belonging to groups 3 and 4, and group indices of respectively 3.78 and 4.05. The modulation sections occupy about $60 \%$ of the ring's circumferences, while the rest of the circumferences are used to implement thermal tuning sections in order to control the resonance wavelength of the rings (note that the resulting performance reduction could have been avoided by implementing the thermal tuners in an additional heater layer above the rings). Each group of modulators (Tx 1-4) is formed by four single RRMs sharing the same through port, but each have their individual drop port taps in order to monitor their resonance frequency. For each of the four rings within a given group, a slightly different waveguide coupling factor $\kappa$ was chosen which results in different Q-factors. Groups Tx 1 and Tx 4 consist of over-coupled rings, whereas groups Tx 2 and 3 consist of under-coupled rings. In fig. 7 the optical modulation amplitude (OMA), defined as $O M A=\frac{P_{1}-P_{0}}{P_{\text {in }}}$ where $P_{\text {in }}$ is the input power inside the bus waveguide, $P_{1}$ is the optical power transmitted in the onstate, and $P_{0}$ is the optical power transmitted in the off-state, is plotted against the measured electro-optic bandwidth for the four groups of rings. Tx 1 and 2 are combined as $46 \mu \mathrm{m}$ rings and Tx 3 and 4 as $28 \mu \mathrm{m}$ rings. The applied bias voltage was set to $-0.5 \mathrm{~V}$ and the peak-to-peak voltage was set to $2 \mathrm{~V}$. For the measurement an Agilent $50 \mathrm{GHz} \mathrm{VNA}$ and a U2T/Finisar high-speed photo-receiver (40 GHz bandwidth XPRV2021A) were used. Both OMA and bandwidth were measured for a laser detuning corresponding to maximum DC response.
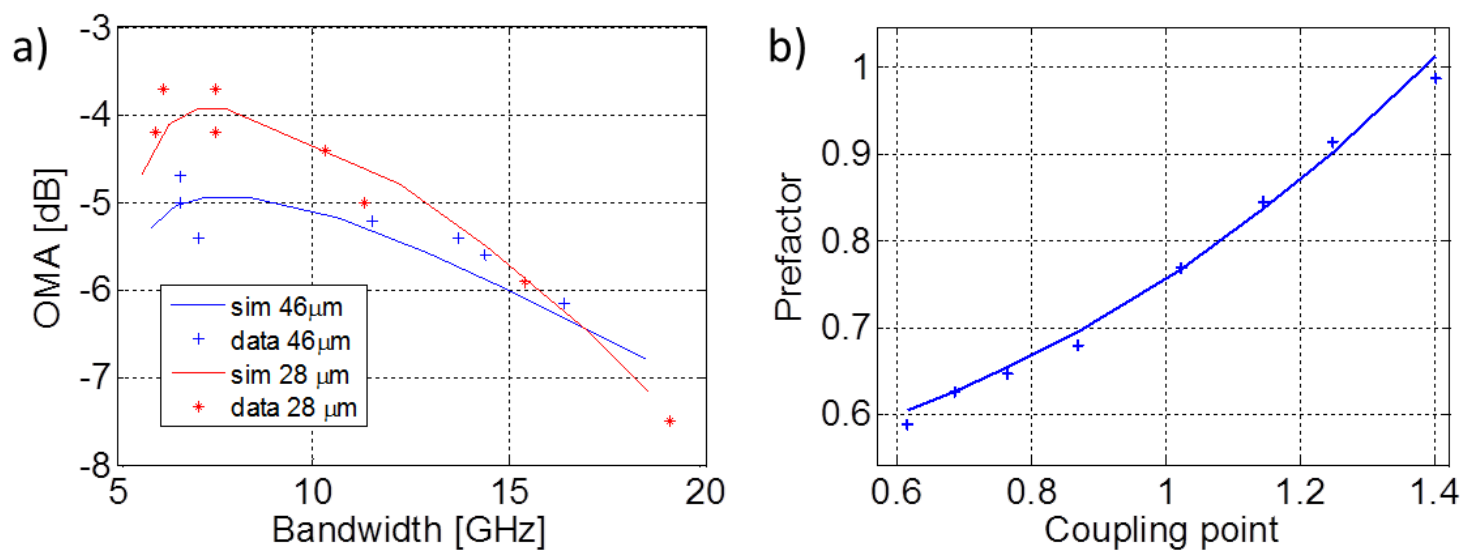

Fig. 7: (a) Tradeoff between OMA and modulation bandwidth for different ring designs compared with simulation results (solid lines). The intrinsic Q-factor is the same for all the rings of a given curve. Designs are varied by changing the coupling parameter to the bus waveguide. (b) Prefactor for bandwidth calculations extracted from eq. 6 for different coupling points $\kappa / \kappa_{0}$, where $\kappa$ is the implemented amplitude coupling factor between the ring and the bus waveguide and $\kappa_{0}$ is the coupling factor that would have resulted in critical coupling. 
We also simulated the OMA and the cut-off frequency by solving the diode equations and calculating the overlap integral of the waveguide with the depletion width. From these equations we obtained $\Delta \mathrm{n}_{\text {eff }}$ of 7.6e-5 for Tx 1 and 2 , and a $\Delta \mathrm{n}_{\text {eff }}$ of $9.45 \mathrm{e}-5$ for Tx 3 and 4 . The cut-off frequencies arising from the RC time constant were extracted to be 30.8 $\mathrm{GHz}$ and $23.5 \mathrm{GHz}$ respectively. The difference in modulation efficiency between the two designs results from the different mode confinements inside the waveguide (fig. 8). For the shallow etched, wider waveguides we get less overlap of the mode with the depletion zone, but at the same time we get a lower series resistance and as a consequence a higher electrical (RC limited) cut-off frequency. For the deeper etched, narrower waveguides the overlap is higher, but the series resistance is increased and leads to a reduced electrical bandwidth. Therefore, the $28 \mu \mathrm{m}$ rings (fig. 7(a) red curve) have higher modulation efficiency for smaller Q-factors (that lead to bandwidths up to $15 \mathrm{GHz}$ ), but then due to the lower electrical cut-off the $46 \mu \mathrm{m}$ rings (fig. 7(a) blue curve) become more efficient.
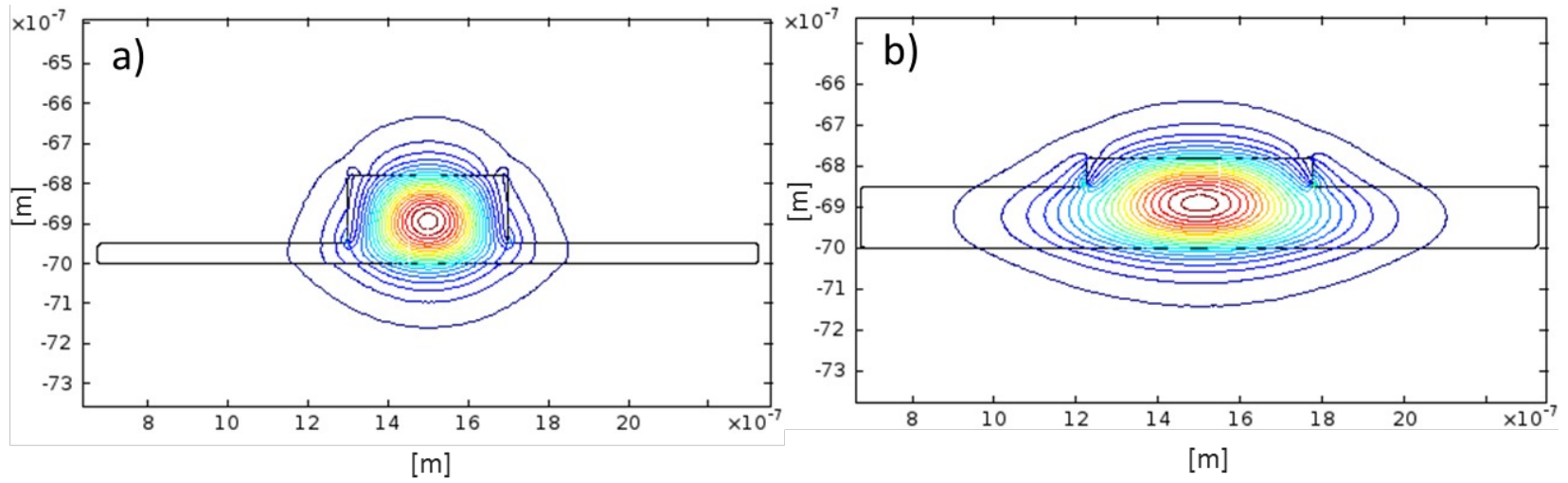

Fig. 8: Mode confinement for (a) deep etched rings with $28 \mu \mathrm{m}$ radius and (b) shallow etched rings with $46 \mu \mathrm{m}$ radius

In order to calculate the optical bandwidth (electrical convention) we used the following formula:

$$
f_{\text {opt }}=D\left(\frac{\kappa}{\kappa_{0}}\right) \frac{f_{r}}{Q_{L}}
$$

where $f_{r}$ is the resonance frequency of the ring resonator, $Q_{L}$ is the loaded Q-factor and $D$ is a prefactor that depends on the coupling point $\kappa / \kappa_{0}$ of the RRM (fig. 7(b)), where $\kappa$ is the implemented amplitude coupling factor between the ring and the bus waveguide and $\kappa_{0}$ is the coupling factor that would have led to critical coupling. Critical coupling corresponds to $\kappa / \kappa_{0}=1$, over-coupling to $\kappa / \kappa_{0}>1$ and under-coupling to $\kappa / \kappa_{0}<1$. The prefactor $D$ was extracted from eq. 6 for the measured $\kappa$ and features a quadratic dependence in the experimentally explored region (fig. 7(b)). As can be seen in fig. 7(a), we obtained a good agreement with our measurements. It should be noted that in the absence of dynamic losses and assuming that only the term in eq. 6 with the closest pole dominates, one would straightforwardly derive a laser detuning for optimum OMA of $f_{r} / 2 \sqrt{3} Q_{L}$ and an optically limited $-3 \mathrm{dBe}$ bandwidth of $(1 / 2+$ $1 / 2 \sqrt{3})\left(f_{r} / Q_{L}\right) \approx 0.79 f_{r} / Q_{L}$. Due to the two aforementioned effects, however, the actual prefactor is slightly different and depends on the coupling point.

Another way of trading off OMA with bandwidth is to use the peaking effect that was described above. Figure 9(a) shows the optical transmission spectrum of a RRM with several optical carrier wavelengths corresponding to the $S_{21}$ measurements shown fig. 9(b). We compared these measurements with eq. 6 with a very good agreement. We fitted the change of photon lifetime versus resonance shift $\vartheta=\partial \tau_{a}{ }^{-1} / \partial \operatorname{Re}\left(\delta \omega_{r}\right)=-\partial \operatorname{Im}\left(\delta \omega_{r}\right) / \partial \operatorname{Re}\left(\delta \omega_{r}\right)$ to be 0.03 . We also had to adjust the assumed laser detunings a few picometers from the expected values due to drift during the experiment. With the exception of these two fitted quantities, the comparison only relies on measured values.

Conventionally, the optimum detuning frequency is defined as the laser detuning for which the OMA is maximized. There is, however, also another figure of merit consisting in the product of the OMA (on a linear scale) and the photon lifetime limited (and peaking enhanced) $3 \mathrm{~dB}$ bandwidth (the OBP product). This product stays invariant when the Qfactor of the RRM is changed, provided the waveguide coupling strength and the laser biasing are jointly adjusted (laser detuning adjusted to stay the same in terms of percent of the full width at half maximum (FWHM) of the resonance, coupling strength adjusted to maintain the same coupling point $\kappa / \kappa_{0}$ ). That is, once the OBP is maximized, the Q-factor 
can be adjusted in order to obtain the targeted bandwidth, also resulting in an optimized OMA. This simple scaling law ignores some practical modalities: For example, when changing the Q-factor one might also want to adjust the implant densities thus enhancing the phase shifter efficiency at lower Q-factors. However, it neatly exemplifies that choosing the point of maximum OMA does not necessarily result in the best biasing, since a larger detuning results in a larger OBP. Figure 10 shows the OBP (a) and the OMA (b) versus detuning for a ring of group Tx 1 with a loaded Q-factor of 12900. When comparing the optima we find that the best detuning is at $\Delta \mathrm{f}=4.55 \mathrm{GHz}$ to maximize the OMA and at $\Delta \mathrm{f}=5.44$ $\mathrm{GHz}$ to maximize the OBP. Changing the laser detuning from $4.55 \mathrm{GHz}$ to $5.44 \mathrm{GHz}$ increases the OBP by $\%$ based on the conservative bandwidth definition and by $13 \%$ based on the aggressive bandwidth definition defined below.

a)

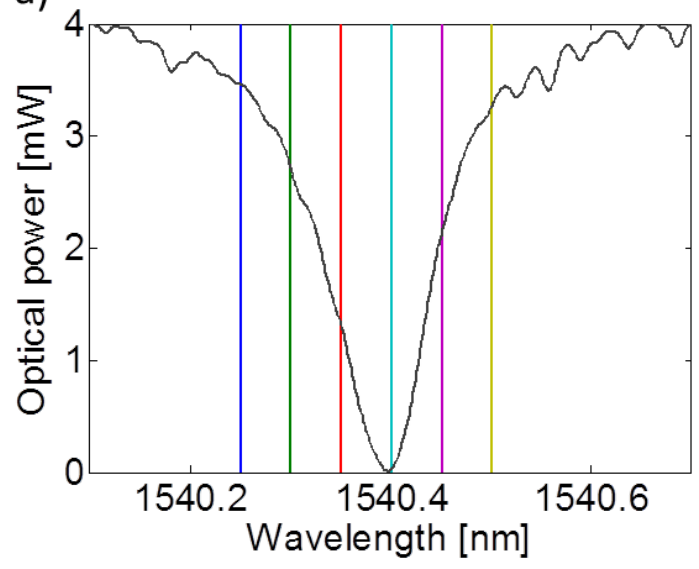

b)

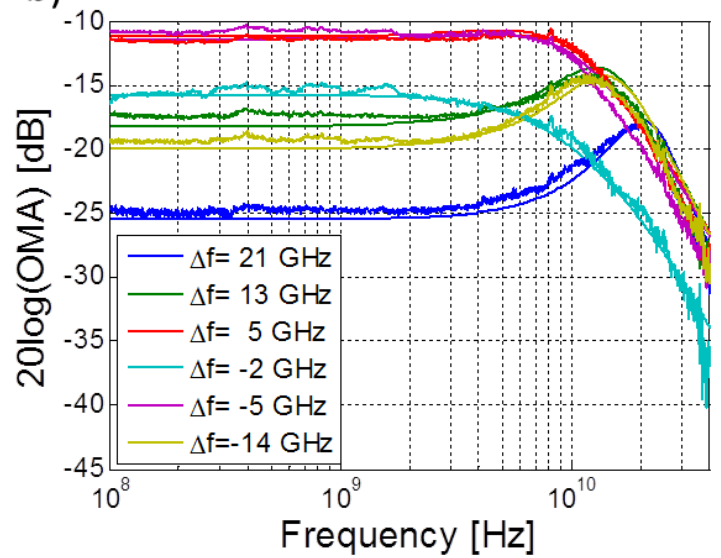

Fig. 9: (a) Optical transmission spectrum with indicated bias wavelengths. (b) $\mathrm{S}_{21}$ measurements for indicated optical carrier wavelengths fitted with eq. 6 .
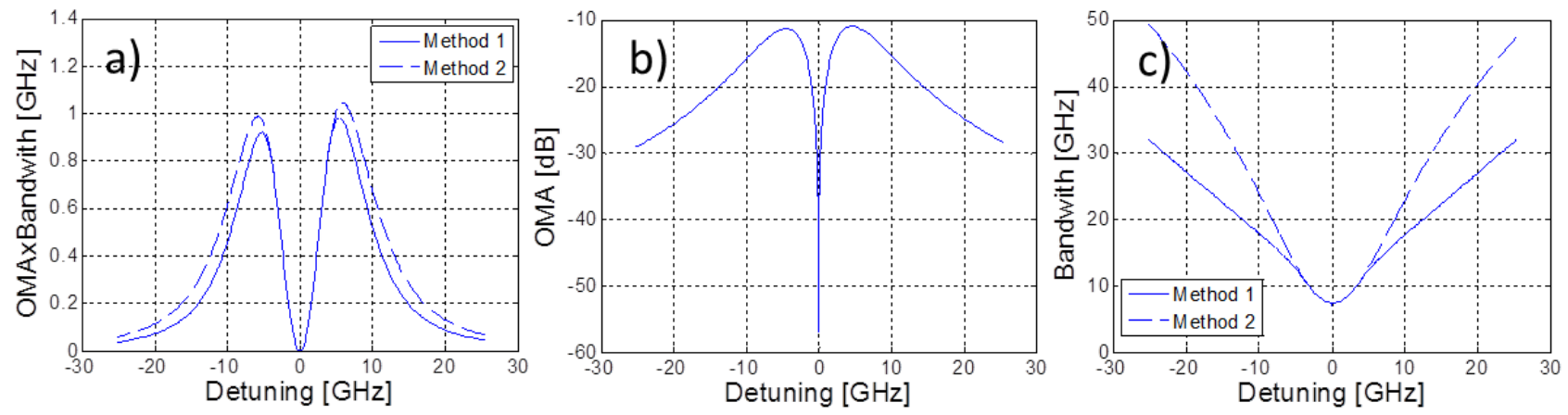

Fig. 10: (a) OMA-bandwidth product (b) OMA and (c) bandwidth over detuning for a selected RRM. The solid curves correspond to a conservative definition of the bandwidth and the dashed curves to an aggressive definition of the bandwidth (described in the text below).

We used two different methods to extract the $-3 \mathrm{dBe}$ electro-optic bandwidth. Method 1 is the more conservative and consists in taking the modulation frequency with the highest $S_{21}$ (the peaking maximum) as the reference for the $-3 \mathrm{dBe}$ cut-off point. Method 2 is more aggressive (i.e., results in higher bandwidths) and takes the DC transmission as a reference. While method 2 is sometimes used in the literature, we believe method 1 to be the fairer metric as a primary source of inter-symbol-interference (ISI) resides in the phase distortion resulting from poles in the $\mathrm{S}_{21}$ transfer function. It can be seen in fig. 10 (c) that the solid line (method 1) shows a lower bandwidth than the dashed line (method 2) when the detuning is high enough to enable peaking (the point of optimum OBP has a slight peaking of $0.4 \mathrm{~dB}$ above DC level for the ring analyzed in fig. 10). Peaking can also be used to enhance the bandwidth after fabrication of a device trading off modulation efficiency, thus dynamically reconfiguring the electro-optical transceiver. 
Figure 11 summarizes highest achievable OMA and highest achievable OBP for respectively optimized laser detuning as a function of the coupling point. As expected, the maximum OMA can be achieved for slightly under-coupled rings (where the Q-factor is slightly enhanced at the cost of a slightly reduced extinction), while the maximum OBP is achieved for slightly over-coupled rings (for which the bandwidth is slightly increased due to Q-spoiling).
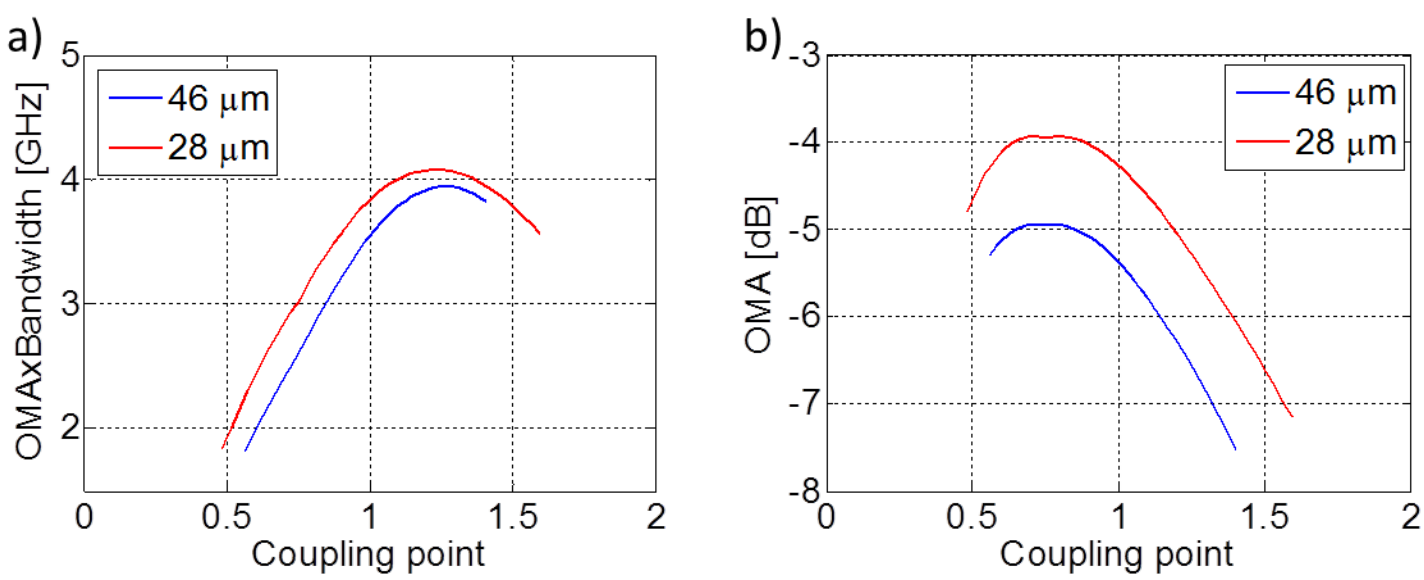

Fig. 11: (a) Simulation of OMA-bandwidth product vs. coupling point and (b) OMA vs. coupling point for both families of rings.

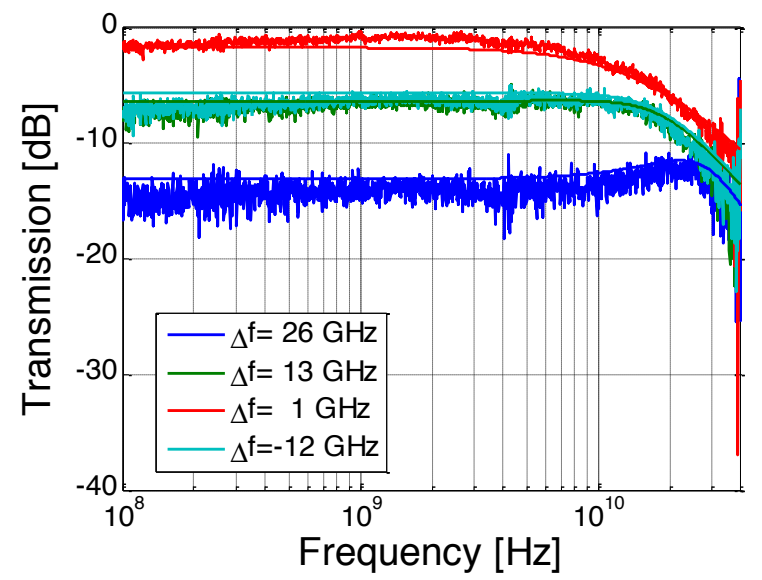

Fig 12: Measured and fitted $\mathrm{S}_{21}$ measurements for the add-drop multiplexer described in the text.

Modeling based on eq. 13 is overlaid on the experimental data.

Bandwidth modification resulting from optical carrier detuning is a phenomenon that can also be seen in add-drop multiplexers (ADM). ADMs are an attractive option for wavelength division multiplexed (WDM) systems due to their small size. Like RRMs, they typically have a tuning section to compensate for the high sensitivity of the resonance frequency to fabrication tolerances and variable ambient temperature [31]. Here, we are going to focus on the description of their behavior depending on the offset of the carrier frequency to the resonance frequency of the ring resonator (detuning). The analyzed ADM was designed similarly to the RRMs from groups Tx 3 and 4, but only the tuning section was doped. The intrinsic Q of the ring is 55000. The coupling factors between the ring and the through and drop ports were designed to be equal and highly over-coupled to achieve a near critical coupling condition with low ADM insertion losses. The extracted amplitude coupling factor $\kappa$ is 0.4 and the loaded Q-factor is 6200 . In order to measure the frequency response of the ADM we used a balanced $40 \mathrm{GHz}$ Mach-Zehnder interferometer operated in push-pull configuration at the $3 \mathrm{~dB}$ point to generate the signal. This signal was then filtered by our ADM and recorded as described above with an Agilent $50 \mathrm{GHz}$ VNA and a U2T/Finisar high-speed photo-receiver. Figure 12 shows the measured $S_{21}$ at resonance as well as for three different detuned frequencies. While, as expected, the RF $S_{21}$ drops, the bandwidth of the device is also increased. We could compare our measurements with the model described in section 3.1 
and obtained a very good agreement between our model and the measurements. It should be noted that while in RRMs bandwidth enhancement via detuning is a practically useful technique since increasing the bandwidth generally requires sacrificing OMA irrespectively of the method chosen, in ADMs it is more of a curiosity since a properly designed ADM should already have the right signal bandwidth. There is one situation however where this may prove to be useful: When communication channels are closely packed such that overlap between the ADM transfer functions and the resulting channel cross-talk becomes an issue, this technique may help alleviating the problem and finding a suitable trade-off.

\section{CONCLUSIONS}

Simultaneously increasing the bandwidth and the modulation efficiency of Silicon Photonics electro-optic modulators is a challenge that also constitutes the focus of the work presented in this paper. Segmented electrodes allow circumventing some of the limitations of coplanar transmission line designs for linear travelling wave Silicon Photonic modulators in simple single layer or double layer interconnect back-end processes. In particular they facilitate impedance matching in modulators with highly doped phase tuners as well as phase matching in modulators with lower doped phase tuners. A travelling wave modulator with a dual drive $50 \Omega$ matched input was demonstrated with full extinction at $3.2 \mathrm{~V}$ signal level, $6.5 \mathrm{~dB}$ on-chip insertion losses and a $17 \mathrm{GHz}$ electro-optic bandwidth. Open $32 \mathrm{Gbps}$ eye diagrams were demonstrated with $2 \mathrm{~V}$ signal levels and $10 \mathrm{~mW}$ laser power in the absence of optical amplification even though high cumulative fiber-to-chip losses of $9 \mathrm{~dB}$ were incurred due to non-optimized grating couplers. Time dynamics in resonant devices do not only lead to peaking in the electro-optic transfer function of ring resonator modulators, but also to peaking in the signal transfer function of ring based add-drop multiplexers. Both effects were modeled and compared to experiments with excellent agreement. We introduce the optical modulation amplitude - electro-optic bandwidth product as a figure of merit for ring resonator modulators and show that different waveguide coupling points and optical carrier to ring resonance detunings lead to an optimum for the latter than for ring modulators solely optimized for optical modulation amplitude. Dynamically adjusting the optical carrier detuning in resonant ring modulators allows trading-off modulation efficiency with modulation bandwidth and reconfiguring electro-optic transceivers. A $17 \mathrm{GHz}$ ring modulator suitable for $25 \mathrm{Gbps}$ serial communication with a $6.5 \mathrm{~dB}$ modulation penalty when driven with a $2 \mathrm{~V}$ signal is demonstrated.

\section{ACKNOWLEDGEMENTS}

The authors gratefully acknowledge funding by the European Research Council (ERC) for the project "Frontiers of Integrated Silicon Nanophotonics in Telecommunications" under contract no. 279770, by the European Commission for the project "Broadband Integrated and Green Photonic Interconnects for high-Performance computing and Enterprise Systems" (BIG PIPES) under contract no. 619591 and by the German federal ministry of science and education for the project "Safe and Secure European Router" under contract no. 16BP12504. Devices described in this paper were fabricated at IMEC via the shuttle-service EpixFab and at the Singapore Institute of Microelectronics (IME).

\section{REFERENCES}

[1] A. Ghiasi, "Is there a Need for on-Chip Photonic Integration for Large Data Warehouse Switches," Proc. $9^{\text {th }}$ Int. Conf. on Group IV Photonics, 27-29 (2012).

[2] A. Narasimha et al., "An ultra-low power CMOS photonics technology platform for $\mathrm{H} / \mathrm{S}$ optoelectronic transceivers at less than $\$ 1$ per Gbps," Optical Fiber Communication Conference (OFC), paper OMV4 (2010).

[3] A. Mekis et al., "Monolithic integration of photonic and electronic circuits in a CMOS process," Proc. of the SPIE 6897, 68970L (2008).

[4] G. T. Reed, G. Mashanovich, F. Y. Gardes, D. J. Thomson, "Silicon optical modulators," Nature Photonics 4(8), 518-526 (2010).

[5] J. Witzens, G. Masini, S. Sahni, B. Analui, C. Gunn, G. Capellini, "10Gbit/s transceiver on silicon," Proc. of the SPIE 6996, 699610 (2008).

[6] See for example the recently announced 100G CWDM4, CLR4 and PSM4 multi source agreements. 
[7] X. Xiao et al., "High-speed, low-loss silicon Mach-Zehnder modulators with doping optimization," Opt. Express, 21(4), 4116-4125 (2013).

[8] J. C. Rosenberg et al., "A 25 Gbps silicon microring modulator based on an interleaved junction," Opt. Express 20(24), 26411-26423 (2012).

[9] F. Merget, S. S. Azadeh, J. Mueller, B. Shen, M. P. Nezhad, J. Hauck, J. Witzens, "Silicon photonics plasmamodulators with advanced transmission line design," Opt. Express 21(17), 19593-19607 (2013).

[10] D. Kucharski et al., "Distributed amplifier optical modulator," US Patent US7515775.

[11] J. Witzens, T. Baehr-Jones, M. Hochberg, "Design of transmission line driven slot waveguide Mach-Zehnder Interferometers and application to analog optical links," Opt. Express 18(16), 16902-16928 (2010).

[12] H. Yu, W. Bogaerts, "An Equivalent Circuit Model of the Traveling Wave Electrode for Carrier-Depletion-Based Silicon Optical Modulators," J. Lightwave Tech. 30(11), 1602-1609 (2012).

[13] D. A. B. Miller, "Energy consumption in optical modulators for interconnects," Opt. Express 20(S2), A293-A308 (2012).

[14] Q. Xu, B. Schmidt, S. Pradhan, M. Lipson, "Micrometre-scale silicon electro-optic modulator," Nature 435(7040), 325-327 (2005).

[15] H. C. Nguyen et al., "Compact and fast photonic crystal silicon optical modulators," Opt. Express 20(20), 2246522474 (2012).

[16] G. Li et al., "Ring Resonator Modulators in Silicon for Interchip Photonic Links," J. Sel. Top. Quant. Electron. 19(6), Article 3401819 (2013).

[17] S. R. Sakamoto, R. Spickermann, N. Dagli, "Narrow gap coplanar slow wave electrode for traveling wave electrooptical modulators," Elec. Lett. 31(14), 1183-1185 (1995).

[18] A. F. Jaeger, K. F. Lee, "Slow-Wave Electrode for Use in Compound Semiconductor Electro optic Modulators," IEEE J. Quant. Elec. 28(8), 1778-1783 (1992).

[19] L. Chen, C. R. Doerr, P. Dong, Y.-K. Chen, "Monolithic silicon chip with 10 modulator channels at 25 Gbps and 100-GHz spacing," Opt. Express 19(26), B946-B951 (2011).

[20] R. Ding et al., "High-Speed Silicon Modulator with Slow-Wave Electrodes and Fully Independent Differential Drive," J. Lightwave Tech. 32(12), 2240-2247 (2014).

[21] Q. Xu, B. Schmidt, J. Shakya, M. Lipson, "Cascaded silicon micro-ring modulators for WDM optical interconnection," Opt. Express 14, 9431-9435 (2006).

[22] Q. Xu, S. Manipatruni, B. Schmidt, J. Shakya, M. Lipson, "12.5 Gbit/s carrier-injection-based silicon micro-ring silicon modulators," Opt. Express 15, 430-436 (2007).

[23] X. Xiao, et al., "44-Gb/s Silicon Microring Modulators Based on Zigzag PN Junctions," Phot. Tech. Lett. 24, 17121714 (2012).

[24]H. Yu, D. Ying, M. Pantouvaki, J. Van Campenhout, P. Absil, Y. Hao, J. Yang, X. Jiang, "Trade-off between optical modulation amplitude and modulation bandwidth of silicon micro-ring modulators," Opt. Express 22(12), 15178-15189 (2014).

[25] J. Müller, F. Merget, S. Sharif Azadeh, J. Hauck, S. Romero García, B. Shen, J. Witzens, "Optical Peaking Enhancement in High-Speed Ring Modulators," accepted for publication at Scientific Reports.

[26] J. Müller, F. Merget, S. Sharif Azadeh, J. Hauck, S. Romero García, "Peaking in ring modulators and application to ISI reduction," to be held at the $11^{\text {th }}$ International Conference on Group IV Photonics (GFP), Paris, France, 27-29 August 2014.

[27] B. E. Little, S. T. Chu, H. A. Haus, J. Foresi, J.-P. Laine, "Microring Resonator Channel Dropping Filters," J. Lightwave Tech. 15, 998-1005 (1997).

[28] W. D. Sacher, J. K. S. Poon, "Dynamics of microring resonator modulators," Opt. Express 20, 15741-15753 (2008).

[29] L. Zhang, Y. Li, J.-Y. Yang, M. Song, R. G. Beausoleil, A. E. Willner, "Silicon-based Microring Resonator Modulators for Intensity Modulation,” J. Select. Top. Quant. Elect. 16, 149-158 (2010).

[30] Y. Ban, J.-M. Lee, W.-Y. Choi, "A Small-Signal Model for Modulation Response of a Silicon Ring Modulator," Proc. 10th Int. Conf. on Group IV Photonics, 124-125 (2013).

[31] X. Zheng et al., "A tunable 1x4 silicon CMOS photonic wavelength multiplexer/demultiplexer for dense optical interconnects," Opt. Express 18(5), 5151-5160 (2010). 